Artículo de reflexión

\title{
Transformación de la no-Complejidad a la Complejidad
}

\section{Transformation of non-Complexity to Complexity}

\section{Carlos Eduardo Maldonado ${ }^{1}$}

${ }_{1}^{1}$ Profesor Titular, Facultad de Ciencia Política y Gobierno, Universidad del Rosario.

Correo electrónico: carlos.maldonado@urosario.edu.co

Recibido: 23-11-2015. Modificado: 13-04-2016. Aceptado: 25-07-2016

\section{Resumen}

Contexto: Este artículo aborda un problema nuevo y difícil: la manera como un fenómeno o sistema simple o lineal puede ser transformado gracias a la geometría de fractales en un sistema o fenómeno complejo. En este sentido, el contexto es el de las ciencias de la complejidad. El problema es altamente significativo, pues de manera general siempre se ha dicho que la complejidad trata, entre otros, con comportamientos no-lineales.

Método: El método es eminentemente teórico. En la bibliografía especializada el problema nunca se ha trabajado, y si sí, cómo un sistema simple o complicado puede ser cambiado en uno complejo o no-lineal.

Resultados: Los resultados indican que es posible abordar el problema y resolverlo satisfactoriamente, atendiendo a los más destacados antecedentes en la materia. Se presentan varios argumentos que remiten entre otros a G. Julia y Mandelbrot.

Conclusiones: La conclusión es que la geometría de fractales suministra bases suficientes para estudiar la transformación estudiada. Así, la importancia teórica y práctica del problema considerado puede extenderse a numerosos campos; aquí se explora y se muestra, por primera vez, cómo dicha transformación es posible.

Palabras clave: Cambio, complejidad, geometría de fractales, métodos analíticos, revolución científica.

Idioma: Español

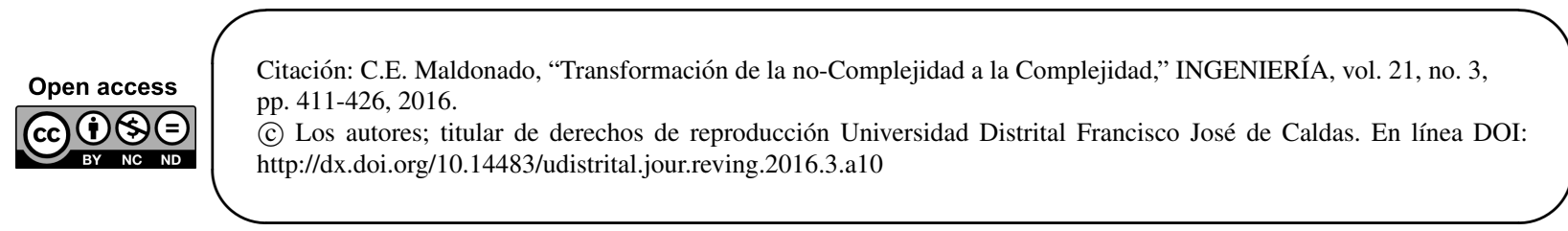

INGENIERÍA • VOL. $21 \bullet$ NO. 3 • ISSN 0121-750X • E-ISSN 2344-8393 • UNIVERSIDAD DISTRITAL FRANCISCO JOSÉ DE CALDAS 


\begin{abstract}
Context: This paper deals with a new and most difficult problem, namely the way in which a simple or linear system or phenomenon can be transformed into a complex or non-linear phenomenon or system thanks to the fractals geometry. In this sense, the framework is set out by the sciences of complexity. Such a problem is extremely important, for in general it has been said that complex science deals, among others, with non-linear behaviors. As a conclusion, the geometry of fractals provides bases solid enough to study the transformation herewith considered. The theoretical and practical meaning of the problem raised here can be extended to numerous fields. Here such a transformation is explored and shown for the first time.

Method: The method here is theoretical. However, in the specialized bibliography the problem considered here has never been worked out, namely: whether, and if so how a simple or complicated system can be changed into a complex or non-linear one.

Results: Working on the basis of fractal geometry the transformation from linear systems into nonlinear is possible. Various arguments are shown that support an idea that sends back to G. Julia and Mandelbrot.

Conclusions: As a conclusion, the geometry of fractals provides bases solid enough to study the transformation herewith considered. The theoretical and practical meaning of the problem raised here can be extended to numerous fields. Here such a transformation is ex- plored and shown for the first time.
\end{abstract}

Keywords: Complexity, fractal geometry, analytical methods, change, scientific revolution

\title{
Preámbulo
}

Cabe distinguir tres clases de sistemas, fenómenos o comportamientos así: sistemas simples, complicados y complejos. Un sistema simple es aquel que puede ser entendido $-\mathrm{y}$ en el orden de la praxis gestionado- en términos agregativos o compositivos. Sin embargo, al mismo tiempo, es simple todo aquello que se puede comprender y manejar en términos de análisis, pues analizar consiste en dividir, compartimentar, fragmentar, segmentar.

Conjuntos de sistemas simples dan lugar a sistemas complicados. En este sentido, entonces, la manera habitual de tratar a los sistemas complicados es en términos, por ejemplo, de distribuciones normales, estadística descriptiva e inferencial, promedios, estándares, matrices y vectores. En el corpus de complejidad (complexity science) es habitual distinguir tal clase de fenómenos o comportamientos. Kuhnianamente hablando los sistemas simples y complicados constituyen ciencia normal.

Otra manera de entender a ambos sistemas es que, en el marco del trabajo e interés por las ciencias de la complejidad, sirven sencillamente como grupo de control. Toda la atención, en contraste, se desplaza hacia el tercer grupo: sistemas, fenómenos o comportamientos complejos. Este artículo trata del tercer grupo a partir de un problema central: comprender cómo los sistemas no-complejos 
- por definición, simples o complicados_- dan lugar, o pueden ser transformados en sistemas complejos en el sentido propio de la palabra. Como es sabido, no existe una única definición de complejidad y la buena ciencia no parte de, ni trabaja tampoco con definiciones. La manera habitual de comprender a un sistema complejo es por sus atributos o propiedades; entre otros, no-linealidad, emergencia, autoorganización, turbulencias, fluctuaciones, comportamiento colectivo complejo y adaptación.

\section{Introducción}

Los dos problemas fundamentales de la complejidad son ${ }^{1}$ : el tiempo y la no-linealidad. Presento la expresión "los dos problemas fundamentales de la complejidad" en analogía al estudio clásico de K. Popper - Los dos problemas fundamentales de la epistemología, redactado originalmente en 1930-33-, solo que el contexto y los problemas son diferentes. Mientras que para Popper los dos problemas fundamentales de la epistemología eran el problema de la inducción y el criterio de demarcación, para nosotros el tema es el de las ciencias de la complejidad. Vale recordar que Popper logra resolver el primer problema pero deja abierto el segundo.

Pues bien, el primer problema de la complejidad, el tiempo, constituye el motivo central de la primera de las ciencias de la complejidad ${ }^{2}$ a saber: la termodinámica del no-equilibrio; pero en el corpus de los clásicos de la complejidad termina en la exploración y formulación de la cuarta ley de la termodinámica por parte de S. Kauffman [1]. Al fin y al cabo, por ejemplo cuando en 1977 la Academia de Ciencias de Noruega le confiere el Premio Nobel a I. Prigogine especifica: "Prigogine introdujo en la ciencia lo que la ciencia no tenía: tiempo, historia". Toda la obra de Prigogine pivota, notablemente, en torno a la importancia del tiempo: desde sus estudios sobre la física del ser a la física del devenir, la discusión sobre si el tiempo es o no una ilusión, como lo sostenía Einstein, y su importancia en la metamorfosis de la ciencia (léase, revolución científica). Un espacio al primer problema fundamental de la complejidad se encuentra en [2], [3].

Así pues, quiero plantear que el segundo problema fundamental de la complejidad consiste, sin lugar a dudas, en el estudio acerca de si y cómo la linealidad se transforma o puede transformarse en no-linealidad. Este texto se ocupa de dicho problema y contribuye a resolverlo, que es malgré Prigogine, el más importante y difícil. A continuación, de manera inmediata, este texto aborda la elucidación del enunciado que se acaba de introducir. En este sentido, el núcleo apunta hacia la geometría de fractales como a la instancia en la cual y gracias a la cual es posible resolver el segundo problema mencionado.

A fin de estudiar este problema, este texto se articula en cinco partes, así: en un primer momento se aborda la importancia de la fenomenología en la complejidad, algo que es particularmente im-

\footnotetext{
${ }^{1}$ Este artículo forma parte del proyecto de investigación "Complejidad y ciencias sociales”, financiado por el CEPI de la Facultad de Ciencia Política y Gobierno de la Universidad del Rosario.

${ }^{2}$ La termodinámica del no-equilibrio es cronológica o históricamente hablando la primera de las ciencias de la complejidad, algo que, a su manera, pone en claro L. Margulis (What is Life?). Las ciencias de la complejidad se componen, a mi modo de ver, de la termodinámica del no-equilibrio, el caos, los fractales, la teoría de catástrofes, la vida artificial, las redes complejas y las lógicas no-clásicas [3].
} 
portante en Prigogine y en Mandelbrot; en segunda instancia, el argumento gira hacia la topología y los fractales, mostrando cómo la geometría de fractales constituye un paso radical hacia adelante relativamente a la topología; gracias a este segundo argumento es posible sostener, en tercer lugar,

la historia del pensamiento fractal, tanto a la luz de lo expuesto por el propio B. Mandelbrot, como por parte de la historia de la matemática y la lógica; el cuarto argumento destaca la importancia de los fractales para pensar patrones, pautas (patterns); al final se elaboran unas conclusiones generales y el texto termina con una coda, en estrecha relación con lo tratado, abriendo la referencia, o complementándola, en otra dirección.

\section{2. ¿Por qué razón la no-linealidad se revela como más impor- tante que el tiempo?}

En la comunidad de complejólogos hace rato que el problema del tiempo ha sido reconocido como fundamental (Kauffman, Solé, Goodwin, y muchos otros). De hecho, sin duda, el primero de los rasgos que abre de manera definitiva la puerta a la consolidación de las ciencias de la complejidad como una novedosa inflexión en la historia de la ciencia [4] es el tiempo. De manera puntual, se trata, en contraste con el papel negativo que le asigna al tiempo la termodinámica clásica y más específicamente el segundo principio de la entropía, del reconocimiento del papel creador o generador de estructuras de complejidad creciente por parte del tiempo. Anticipado por Darwin, este papel creador del tiempo es el resultado de la obra de Prigogine y de lo que ha llegado a conocerse como la termodinámica del no-equilibrio. Dicho sin más, en complejidad el tiempo: a) no es una variable y b) es (lo que determina) la complejidad misma de un fenómeno o sistema.

En contraste con el tiempo, la no-linealidad se encuentra lejos de ser un problema evidente o resuelto, incluso en buena parte de la comunidad de complejólogos. Parcialmente, esto se debe porque no existe una única comprensión de la no-linealidad, si bien sí hay un espacio amplio gracias al cual es posible explicarla y entenderla. Sin embargo, la principal dificultad con respecto a la nolinealidad estriba en el hecho de que en la ciencia normal —en el sentido más amplio e incluyente de la palabra - existen métodos analíticos de tratar y de trabajar con sistemas y fenómenos nolineales. En rigor, estos métodos consisten en una linealización de la no-linealidad. Los ejemplos más conspicuos son: sistemas estocásticos, análisis numérico, constante de Liapunov y sistemas L (de Lindenmeier), aunque puede mencionarse, sin dificultad alguna, la asunción habitual de quienes trabajan en modelamiento y simulación de sistemas complejos, en una amplia mayoría, optan de entrada por parametrizar las dinámicas no-lineales, echando así por la borda la no-linealidad. De hecho, campos vecinos a la complejidad como la cibernética en general, y la cibernética de segundo y de tercer orden en particular, al igual que los enfoques sistémicos le hacen el juego a la ciencia normal en esta dirección o, lo que es equivalente, le hacen un flaco favor a la comprensión de la complejidad misma de la no-linealidad, puesto que, en el mejor de los casos la asumen como una cuestión de lenguaje (semantics).

En propiedad, en complejidad se debe hablar de la no-linealidad de fenómenos y sistemas complejos. En otras palabras, la no-linealidad es un atributo propio de la complejidad. De esta suerte se apunta al no-reduccionismo y no linealización de la no-linealidad. Hay autores que incluso, debido 
a esta observación prefieren hablar no de ciencias de la complejidad sino de ciencias no-lineales, a saber: exactamente en el sentido de la no linealización de la no-linealidad [5].

La clave mediante la cual es posible entender el carácter fundamental de la no-linealidad en complejidad estriba en el reconocimiento explícito de que un fenómeno no-lineal se caracteriza porque gana información (aunque no necesariamente memoria). Así, la no-linealidad y la ganancia de información son mutuamente complementarios. Naturalmente que otras caracterizaciones de la no-linealidad han sido posibles, pero para efectos de este texto, baste con la comprensión señalada: la no-linealidad es información ganada y así, si se prefiere, una no proporcionalidad entre causa(s) $\mathrm{y}$ efecto(s).

Digámoslo de manera franca: el problema medular de la complejidad que aquí nos interesa es el de la(s) transformación(es) de la linealidad en no linealidad. Más exactamente, el problema consiste en lo siguiente: se trata de determinar:

Si, cuándo y cómo un sistema lineal se convierte en uno no-lineal; o bien,

Si, cuándo y cómo un sistema lineal puede ser transformado en uno no-lineal.

En el primer caso, se trataría de estudiar cómo sucede, digamos física u objetivamente, que un sistema determinado rompe la/su linealidad y se torna en un fenómeno no-lineal. Las contribuciones más clásicas al respecto provienen del caos y, más recientemente, de las redes complejas, por ejemplo gracias al estudio de los fenómenos de percolación o las cascadas de errores. El estudio de los solitones, por ejemplo [5] y la producción o emergencia de tsunamis constituye un caso específico. No es este mi interés aquí. Tanto menos cuanto que, en el mejor de los casos, la explicación de esta clase de fenómenos o de situaciones se explica o se entiende ex post.

Por el contrario, me propongo dirigir la mirada hacia el segundo de los casos mencionados, esto es, establecer si y cómo un sistema lineal puede ser transformado en uno no-lineal, ello independientemente de si el agente que lleva a cabo dicha transformación es humano, natural o artificial. La transformación de un fenómeno lineal en uno no-lineal es, en términos matemáticos, una transformación geométrica, pero en términos prácticos se traduce en planos apasionantes como la política o la sociología y que deben, sin embargo, quedar aquí de lado por motivos de espacio.

Mientras que la geometría euclidiana es una abstracción humana cuyo resultado consiste en negar la naturaleza e imponerle formas rígidas, polígonos regulares y construcciones antinaturales, la geometría de fractales corresponde a una naturalización de la geometría. Asistimos así al tránsito de una antropologización de la geometría (= Euclides), esto es, del espacio y por tanto del mundo y la naturaleza, a una naturalización de los mismos (Mandelbrot). El resultado es una revolución magnífica en dos planos.

En verdad, de un lado, se trata del tránsito de un modelo continuo del espacio, el mundo y la naturaleza, a una comprensión discreta de los mismos. Así, la búsqueda e identificación de patrones se corresponde, plano por plano con el descubrimiento de las matemáticas discretas o, lo que es equivalente, de las matemáticas de sistemas discretos. Con seguridad, los componentes centrales de 
esta clase de matemáticas comprenden los teselados, conjuntos extremos, conjuntos parcialmente ordenados, enumeración, teoría de redes, grafos e hipergrafos y la teoría de la codificación.

De otra parte, al mismo tiempo, la búsqueda de patrones abre una perspectiva más natural, o naturalista si se prefiere, de la geometría y, por tanto, del estudio y comprensión del espacio. El resultado habrá de ser el re-descubrimiento de la armonía como un criterio científico y, con ella y más allá de ella, el redescubrimiento de la belleza como un criterio mismo de verdad y validez [6].

\section{Complejidad y fenomenología, un nexo sólido}

Las relaciones entre la fenomenología, como método científico y las ciencias de la complejidad en general es sólido, si bien no muy extendido. En diversas ocasiones, I. Prigogine menciona la importancia de las descripciones fenomenológicas en el desarrollo de sus investigaciones (cfr. La nueva alianza, El nacimiento del tiempo y el libro escrito conjuntamente con G. Nicholis, La estructura de lo complejo $)^{3}$. Algo similar puede evidenciarse en algunos pasajes de la obra de Mandelbrot. Ello, sin embargo, no significa que la complejidad se funde en métodos y aproximaciones fenomenológicos como quisieran considerarlo filósofos fenomenólogos que sí trabajan sobre ese fundamento en otras áreas del conocimiento [7]; ${ }^{4}$ es decir, a la fenomenología en el sentido ya sea de Husserl, Heidegger, Merleau-Ponty o Levinas, para mencionar los nombres más destacados.

En cualquier caso, en el mejor espíritu de la fenomenología como método científico y filosófico el problema de base se formula en los siguientes términos: se trata de distinguir si vemos lo que conocemos o bien si conocemos lo que vemos, un problema que se encuentra en la médula del espíritu, actitud y métodos de la fenomenología en toda la línea de la palabra.

El problema no es trivial, y consiste en clarificar si lo nuevo visto en el mundo se reduce a lo ya conocido y se explica por tanto en términos de lenguajes y experiencias ya adquiridas, o bien si, por el contrario, el investigador se da a la tarea de ver verdaderamente lo nuevo e intentar comprenderlo sin reducirlo a juicios y conceptos ya establecidos. Una ilustración de este problema, sin hacer referencia explícita al cuerpo de la fenomenología, se encuentra en S. Kauffman cuando recuerda la experiencia de los aztecas en su encuentro con los españoles [1] (dicha historia y su significado ha sido estudiada numerosas veces, una muy afortunada referencia es T. Todorov, [17]).

Como se entenderá fácilmente a la luz de una mirada reflexiva, el problema concierne a la capa-

\footnotetext{
${ }^{3}$ De manera puntual, hablamos de fenómeno complejo, fenómeno irreversible, fenómenos de intercambio, fenómenos de regulación, fenómenos de relajación, por ejemplo. Digamos, por lo demás, que en el libro con Nicholis, Prigogine prefiere hablar de fenómenos, sistemas y dinámicas complejos en lugar de hacer referencia, genéricamente, de "ciencias de la complejidad", una expresión que se acuña en el Instituto Santa Fe, en Nuevo México. Parte de la explicación por esta elección por parte de Prigogine está una disputa que tuvo con M. Gell-Mann, pero sobre la cual no cabe aquí hablar.

${ }^{4}$ Dejo aquí de lado cualquier consideración acerca de discusiones más técnicas al interior de la escuelas fenomenológicas, tales como referencias a la fenomenología husserliana o de corte heideggeriano, a la fenomenología francesa del tipo de M. Merleau-Ponty o de E. Lévinas, o acaso también a aproximaciones anglosajonas como las de R. Sokolowski y D. Ihde, B. Benson, entre otros.
} 
cidad de visualización, enfrentamiento y explicación de novedad supuesta la tendencia, natural, a explicar lo nuevo en términos usualmente de analogía o comparación con lo ya conocido.

Pues bien, existe un problema análogo en ciencia. Se trata, expresado, por ejemplo por Einstein, de la disyunción acerca de si pensamos en/con palabras (= signos), o bien en/con diagramas (= símbolos). En el primer caso, puede decirse sin dificultad que se trata de un tipo de pensamiento que favorece aspectos analíticos, en tanto que en el segundo caso se trataría de un acercamiento más visual o sintético a problemas, situaciones, planos del mundo o de la naturaleza.

El padre de la geometría de fractales se inscribe exactamente en este segundo plano y, a través suyo, resuelve el primer problema formulado. En efecto, Mandelbrot expresa en varios lugares que él mismo piensa en términos de diagramas o esquemas y que favorece este tipo de razonamiento sobre el estrictamente analítico, o basado en signos. Extrapolando, y en referencia a la filosofía de las matemáticas en su interpretación más clásica, puede decirse que la geometría de fractales se sitúa, así, más cerca de las tesis intuicionistas (H. Poincaré, Brown) que de las estrictamente formalistas (D. Hilbert). En verdad, el trabajo con fractales es, de plano a plano, el estudio, investigación y disfrute de formas, diagramas, estructuras y gráficos que, si bien, tienen una formulación lógica y matemática, se despliegan ante todo como juegos visuales de gran significado en diversos planos del conocimiento. Gracias a los fractales aprendemos otro tipo de transformación del espacio que el que había sido habitual con Euclides.

Como es sabido, una especificidad de la geometría de fractales es que lleva a cabo operaciones especiales con el espacio. Ya no, como en el caso de Euclides, traslación, reflexión y rotación, y tampoco simplemente como en la topología: torcer, estirar, comprimir. La geometría de fractales lleva a cabo un tercer tipo de operaciones: iteraciones. Ahora bien, en rigor, cabe distinguir dos clases de iteraciones: lineales y no-lineales. Manifiestamente que la iteración constituye un método de resolución de sistemas lineales. Sin embargo, de otro lado, existen igualmente las iteraciones fractales no-lineales cuyo resultado ni es simple, ni complicado y manifiestamente no lineal. Volveré sobre este asunto en la cuarta sección.

\section{Topología y fractales}

La geometría de fractales se encuentra, en sus orígenes, en algún lugar intermedio entre la topología y la geometría. La historia y la importancia de la geometría coinciden, plano por plano, con la historia misma de la humanidad occidental y, más radicalmente, con la historia misma del espíritu humano. Tanto más cuanto que la geometría es la ciencia del espacio, el estudio del espacio y más allá de las discusiones entre físicos y matemáticos acerca de si la geometría pertenece al dominio de unos o de otros.

Como es sabido, grosso modo, la historia de la geometría tiene dos grandes capítulos: la geometría euclidiana, formulada originariamente en el libro Elementos de Euclides y, a raíz de las discusiones sobre el quinto postulado de Euclides, el desarrollo de las geometrías llamadas no-euclidianas, que nacen en el curso del siglo XIX. Esto es, la geometría de Riemann y de Lobachevsky, con las contribuciones de Bolyai. Solo que, gracias al surgimiento de las geometrías no euclidianas, entonces 
se abren tres capítulos nuevos: los fractales, el hiperespacio y la teoría de cuerdas [8]. Dejaremos aquí por razones de delimitación de nuestro tema las referencias al hiperespacio y a la teoría de cuerdas [18]. Dejo aquí de lado las relaciones y distinciones entre la topología estructural y la algebraica, topología de redes, topología dimensional y otros debido a la tesis que define el carácter de este texto.

Por su parte, de manera sucinta, con el nacimiento de las geometrías no-euclidianas, la topología contemporánea se inicia con los trabajos de Euler, pero alcanza su pleno reconocimiento mucho tiempo después, particularmente gracias a los trabajos de S. Smale. Es indudable, sin embargo, que la topología constituye un capítulo fundamental, propio, al interior de la geometría.

El trabajo de la geometría tanto como de la topología coincide en un punto, a saber: en el trabajo con transformaciones en el espacio; transformaciones geométricas, en un caso, y transformaciones topológicas, en el otro. Es fundamental atender a que, particularmente en el caso de la topología, no debe haber rupturas en el espacio, esto es, en, durante o después de las transformaciones operados en la topología. Esta idea permite precisar un concepto general: la geometría es la ciencia del espacio, como la ciencia de las transformaciones en/del espacio, en las que no tienen lugar rupturas del espacio. Estrictamente, se trata de ciencia(s) de sistemas continuos.

Pues bien, en el mismo y en el mejor espíritu de la geometría, el estudio de los fractales también consiste en el estudio de transformaciones geométricas. Más específicamente, las transformaciones que llevan a cabo los fractales son iteraciones. A continuación me ocuparé con mayor detalle de las mismas; su significado estriba en el hecho de que, en contraste con las operaciones de la geometría euclidiana y de la topología, permite acceder, por vía directa, al problema y su resolución de las relaciones entre linealidad y no-linealidad. O bien, como cabe decirlo igualmente, en la transformación de lo no- complejo a la complejidad.

Antes de continuar se impone un pequeño paréntesis histórico. Se trata de esbozar rápidamente los trazos más importantes de los antecedentes y la anatomía de la geometría de fractales. Dicho esbozo es, sin embargo, de gran ayuda, para entender el significado real de las transformaciones fractales que, como lo digo, permiten la transformación de la linealidad en no-linealidad, o bien, lo que es equivalente, de la no-complejidad en complejidad.

\subsection{Breve historia del pensamiento fractal}

La mejor fuente acerca de los antecedentes del pensamiento fractal ha sido elaborada por B. Mandelbrot, notablemente en los capítulos 40-42 de La geometría fractal de la naturaleza: "De los hombres e ideas". Pero este breve recuento histórico se complementa con el "Epílogo" (capítulo 42) en el que resume su propia trayectoria, que habría de conducir a Mandelbrot hasta la redacción y publicación del libro en 1977, marcando una inflexión fundamental en la historia de la ciencia y, por consiguiente, en la historia de la humanidad occidental.

El principio filosófico fundamental de la geometría de fractales es el mismo que el de las transformaciones geométricas en general ya mencionadas, a saber: el principio de continuidad, que se expresa en la fórmula clásica: Natura non facit saltus. Desde este punto de vista, los fractales, en 
la visión del propio Mandelbrot, se sitúan en una historia que une a Aristóteles con Leibniz y a Euclides con Cantor.

En consecuencia, la geometría de fractales quiere exponer una idea contundente: existe un orden en la naturaleza que, sin embargo, no es numerable. En otras palabras, se trata de un orden con respecto al cual no cabe una demostración en el sentido tradicional, axiomático, deductivo, por tanto descendiente, de la palabra. Este orden se expresa adecuadamente en términos de grupos y de cuerpos o relaciones conmutativos que, con la ayuda del computador, se manifiestan ulteriormente en términos de simetrías dinámicas. Eso son los conjuntos de Mandelbrot. La ayuda del computador es determinante en un dúplice sentido: de un lado, se trata de la puerta de entrada a las matemáticas de sistemas discretos; y de otra parte, al mismo tiempo, del basamento mismo de las ciencias de la complejidad.

Desde el punto de vista filosófico (= filosofía de las matemáticas), los números reales aparecen como números racionales e irracionales con lo cual se quiere atender a una idea clara: asistimos al nacimiento de una nueva dimensión en la naturaleza que no había sido considerada anteriormente en la historia de la humanidad. A mi modo de ver, la idea de continuidad es más el resultado de una interpretación que le debe mucho al pasado, antes que de un reconocimiento acerca de la terra incognita que se abre con la geometría de los fractales. ${ }^{5}$

Dos elementos importantes adicionales en los que se incuba la geometría de fractales son la distribución de Gibbs y la invariancia de cambios de escala. La primera permite derivar todas las relaciones termodinámicas clásicas y cuánticas, algo que no tiene una repercusión inmediata en el estudio de los fractales, pero sí para dirigir la mirada hacia los cambios de escala en el uso y estudio de la funciones; la segunda, por su parte, se ocupa de la ausencia de cambios en la escala de tamaño o en la escala de energía. Pues bien, cuando se estudian los fenómenos fractales y en particular cuando se pone claramente sobre la mesa a plena luz del día la noción de dimensión fractal, observamos justamente las estructuras fractales gracias a las cuales la escala de la parte se corresponde (¡fractalmente!) con la escala del todo.

Lo que debe quedar en claro es que la distribución de probabilidad que caracteriza a los fractales es hiperbólica. El estudio de los fractales está lleno de leyes de potencia y, sin la menor duda, la presencia de leyes de potencia constituye un rasgo distintivo de la existencia o la presencia de un sistemas, un fenómeno o un comportamiento complejo.

No en vano, entre los antecedentes más directos de los fractales está el trabajo de Zipf, la ley de Zipf, que la comunidad de complejólogos tan solo acogerá ampliamente a partir de los trabajos de P. Bak [9].

\footnotetext{
${ }^{5}$ En verdad, como lo mostraré a continuación, la geometría de fractales permite abrir la puerta y cruzar el umbral que conduce hacia los sistemas discretos y con ellos a las matemáticas discretas. Quizás el temor al rechazo por parte de la comunidad científica acerca de sus ideas — algo de lo que habrá de lamentarse incluso veinte años después Mandelbrot-, lo obliga a mirar en la dirección de la continuidad. Aunque, de otra parte, es evidente que cuando Mandelbrot publica en 1977 el texto fundamental sobre los fractales, las matemáticas discretas aún no habían alcanzado un derecho al voto propio o licencia de conducción, por así decirlo.
} 
En cualquier caso, como lo observa Mandelbrot a propósito del libro de 1977, se trata de escritos que "empiezan sin prólogo y acaban sin conclusiones" [19]. "Hoy en día, los casos en que técnicas y conceptos nuevos entran en la ciencia a través de ramas poco competitivas son raros, y por ende anómalos. La geometría fractal es un ejemplo más de tal anomalía histórica” [10].

\subsection{Pensar en patrones}

Pensar en sistemas, fenómenos y comportamientos complejos corresponde a identificar patrones (patterns) (aunque no únicamente). Y precisamente por ello, en este marco, equivale a pensar en términos de conjuntos; así, el marco es, genéricamente, la geometría. Sin embargo, la búsqueda de patrones no es exclusiva de los trabajos en torno a complejidad. Un antecedente notable es el trabajo de G. Bateson [11] en torno a "la pauta que conecta" (the conneting pattern); igualmente, Bateson es un autor que nada tiene que ver con complejidad. La ciencia clásica, en contraste, consistió esencialmente en la búsqueda de leyes (laws) que marca un espíritu fisicalista en ciencia e investigación.

En rigor, el desarrollo de y el trabajo con patrones constituye el mérito de la lógica y de la matemática de finales del siglo XIX y comienzos de siglo XX. Antes, la ciencia en general estaba marcada por la idea de leyes y legalidad, de objetos y a lo sumo de relaciones entre ellos. Sin duda, el paradigma es el de la mecánica clásica con Newton y su epítome.

Los nombres que inauguran la tradición que pivota en torno a la búsqueda e identificación de patrones son, con seguridad, Cantor, Peano, Koch, Klein, Julia, todos nombres que se encuentran entre los antecedentes, directos o indirectos de la geometría de fractales. En consecuencia, una observación puntal se impone: una cosa es una iteración de Peano o Cantor, por ejemplo, y otra muy distinta la de Mandelbrot. Aquella es lineal, en tanto que su mérito consiste en haber subrayado el papel de las iteraciones, cuyo resultado es la fractalidad misma. Digámoslo de forma directa: la fractalidad constituye otro de los rasgos distintivos de un sistema complejo.

La idea que emerge inmediatamente es la del trabajo con conjuntos como el trabajo mismo con iteraciones, gracias a las cuales, por lo demás, con Cantor logramos ganar la idea de infinito y, más exactamente, de infinitos infinitos ${ }^{6}$. Como se aprecia, con Cantor, la iteración de una misma operación, o de un mismo patrón, permite al cabo encontrar el infinito (conjuntos infinitos). Por su parte, H. von Koch desarrolla hacia 1904 el famoso conjunto de Koch que corresponde a la geometría de un copo de nieve, el cual es posible igualmente sobre la base de un proceso iterativo. Sin embargo, como quiera que sea, el primero que lleva a cabo un trabajo de transformación del espacio en términos de identificación de patrones fue Möbius en 1858 [12].

Entre los trabajos pioneros de conjuntos que habrían de conducir, ulteriormente, al desarrollo de

\footnotetext{
${ }^{6} \mathrm{El}$ infinito es descubierto o inventado tres veces en la historia de la humanidad y siempre por parte de las matemáticas. La primera vez fue gracias a G. Bruno, en particular en su obra El universo infinito y los mundos, en el siglo XVI. El trabajo de Bruno se plasmó en la astrofísica y la cosmología. El Cardenal Bellarmino (jesuita) juzgó y condenó a la hoguera a Bruno por considerar conceptos contrarios a la Iglesia católica. En efecto, el concepto mismo de "infinito" no aparece en el Libro de los Libros. La segunda vez tuvo lugar gracias a G. Cantor, quien no solamente descubre o inventa el infinito sino, mejor aún, infinitos infinitos. La tercera vez tiene lugar gracias a la geometría de politopos desarrollada por D. Coxeter, entre los años 1930 y 1970.
} 
los fractales y, de manera puntual a las transformaciones realizadas por y en fractales, es indispensable tener en cuenta los antecedentes de Dedekind, Zermelo y Gödel.

El conjunto de Zermelo se expresa en los siguientes términos: $\{\{\{\ldots\}\}\}$

O, lo que es equivalente, así: $\{f(x) \mid x \in A\}$

Como es sabido, la diagonal, de la serie ' $m$ ' y ' $w$ ' muestra la distribución creciente y por tanto la transformación del conjunto en las variaciones $E 0$, etc.

El conjunto de Gödel, por su parte, se expresa en los siguientes términos: $\{x \mid \operatorname{Phi}(x)\}$

Ahora, si se considera un damero, esto es, un plano dividido en casillas se aprecia transformaciones consistentes en traslación, rotación, dilatación e inversión. Es exactamente lo que acontece con una transformación de Möbius.

En cualquier caso, lo que resulta claro a la luz de lo que precede es que el tema fundamental es el espacio, lejos de ser una entidad sólida y rígida, implica, permite, admite transformaciones. Pues bien, la idea que quiero sostener es que las transformaciones del espacio que son, de base, el tema de la geometría, permiten de una manera gradual, si cabe, un desplazamiento de estructuras, formas y sistemas eminentemente lineales hacia dinámicas no-lineales. Este es el tema central de la siguiente sección. Pero antes puntualicemos: las iteraciones de Zermelo, Peano, Dedekind y Cantor son iteraciones fractales lineales y sus resultados son igualmente lineales. Pero algo distinto sucede con Mandelbrot.

\subsection{Patrones y discreción}

El motivo que gatilla en la historia de la geometría el trabajo con la elaboración de patrones y que al cabo conducirá al descubrimiento de la iteración fractal, tiene que ver con la discusiones generadas en torno al quinto postulado de Euclides, en contraste con la aceptación de los primeros cuatro postulados de los Elementos.

Específicamente, la dificultad se encuentra en el hecho de que Euclides plantea las definiciones y los axiomas de la geometría clásica como hechos consumados y sin continuidad entre una dimensión y otra. Sencillamente, es imposible que una línea recta coincida con un plano, o que un plano sea congruente con un sólido, por ejemplo. De manera taxativa: el punto carece de dimensiones, la línea recta es la dimensión uno, el plano es la dimensión dos, el sólido es dimensión tres. Cada dimensión es una estructura lógica y ontológica propia y suficiente.

Con seguridad, la primera transformación radical de los espacios de Euclides es la que llevan a cabo los conjuntos de Peano, que permite ver cómo acontece la transformación de la dimensión uno, en términos euclidianos, en dimensión dos, hasta llenar o convertirse una línea recta en un plano:

Es el momento para formular lo que puede ser considerado como una sub-tesis, relativa al pro- 
blema formulado al comienzo. En contraste con lo que precede, de acuerdo con la idea del mismo Mandelbrot, es que los fractales trabajan, abierta o tácitamente, sobre sistemas continuos, natura non facit saltus; quiero sostener que los sistemas, fenómenos y comportamientos complejos tienen en su base matemáticas discretas. Mejor aún, los sistemas complejos son fenómenos discretos. Sus componentes más destacados pueden ser claramente identificados: conjuntos parcialmente ordenados, conjuntos extremos, geometría discreta y combinatoria, teoría de probabilidades discreta, problemas combinatorios (complejidad combinatoria), teoría de juegos y teoría de la decisión racional, topología, algunas de las lógicas no clásicas y las matemáticas de los sistemas computacionales. La geometría de fractales, contra la interpretación de su propio descubridor, no trata de sistemas continuos, sino de sistemas discretos ${ }^{7}$.

De manera sintomática, pensar en términos de fenómenos, sistemas y dinámicas discretas equivale a pensar en términos de armonía. El estudio de invariancia/simetría se revela como fuente profunda de una armonía común a muchas estructuras. El descubrimiento de la geometría de fractales, por parte de Mandelbrot, ha arrojado nuevas y sugerentes luces en campos diversos como las matemáticas, las finanzas, las ciencias en general, el arte y, de manera puntual, en antropología y arqueología [13].

En efecto, contra el encerramiento del mundo, en términos de patrones y criterios occidentales a partir de los postulados de Euclides, el encuentro de los fractales (notablemente fractales escalantes, multifractales, etc.), puso de manifiesto, con toda claridad, la ubicuidad de la aspereza. En palabras de Mandelbrot: "In one field after another, fractal geometry became the first tool which made it possible to help shape a theory of roughness" [14]. Y más adelante:

Roughness is ubiquitous in Nature. In the works of Man, it may not be welcome, but is not always avoided, and may sometimes be unavoidable. Examples are found in some parts of mathematics, where they were at one time described as 'pathological' or 'monstruous'... [14].

En otras palabras, la geometría de fractales pone de manifiesto que la aspereza en la naturaleza es, paradójicamente cuando se lo observa desde los ojos de la tradición euclidiana, el resultado mismo de la armonía. Pues bien, como es sabido, la aspereza fractal coincide exactamente con, o se funda en, la dimensión fractal.

\section{Iteración fractal como transformación de la linealidad}

La transformación del espacio operada por los fractales es la iteración. Iteraciones, en rigor. Radicalmente, las operaciones de traslación, reflexión y rotación no alteran absolutamente para nada

\footnotetext{
${ }^{7}$ Una observación larga se impone aquí en el espíritu de la filosofía de la ciencia. En fractales, sostengo, sucede algo análogo a lo que vemos con la teoría de la evolución. Darwin interpreta su teoría en términos gradualistas, y Darwin mismo es gradualista a pesar suyo y a pesar de su propia teoría. M. Ruse ha llamado fuertemente la atención en distintos libros sobre este hecho (por ejemplo, The evolution wars: a guide to the debates (2003), Darwin and Design: Does evolution have a purpose? (2003), The Evolution-Creation Struggle (2005), Darwinism and its Discontents (2006)). Más radicalmente, S. J. Gould ha puesto suficientemente de manifiesto que la teoría de la evolución es catastrofista y que Darwin termina siendo gradualista por motivos extra-científicos, a saber: por el peso de la Inglaterra Victoriana.
} 
la linealidad. Otro tanto puede y debe decirse las operaciones llevadas a cabo en y por la topología: torcer, estirar y comprimir, no alteran significativamente la linealidad de un sistema o fenómeno determinados. Las transformaciones operadas en la geometría euclidiana tanto como en la topología corresponden a un pensamiento y ontología de sistemas continuos.

En contraste, las iteraciones fractales corresponden a una ontología discreta y tanto exigen como ponen de manifiesto un modo de pensar específico: pensar en sistemas discretos. Precisamente por ello emergen la aspereza y la armonía. La belleza del universo se naturaliza de manera evidente.

En la base misma de los fractales se encuentra la famosa ecuación: $f(z)=z^{2}+c$

Pues bien, la ecuación productora de fractales consiste exactamente en una iteración mediante la cual accedemos a una noción novedosa en la historia de la geometría, a saber: la idea de equilibrios dinámicos o, lo que es equivalente, la noción de simetrías dinámicas. Sin lugar a duda, el papel cultural, además de científico, del computador, desempeña un rol protagónico, que permite marcar una distancia enorme con respecto a la existencia y desarrollo de patrones e iteraciones, notablemente, en el arte precolombino, desde los Aztecas hasta los Incas, pasando por los Mayas y los Muiscas, por ejemplo. H. Pagels [15] llamó en su momento la atención acerca del papel del computador en el desarrollo de las ciencias de la complejidad y, por extensión, en relación con la forma de pensar qué es la complejidad.

Mandelbrot permaneció casi toda su vida con la preocupación acerca de: a) las deudas con el pasado y b) la novedad que representaban sus propios desarrollos. A los mencionados capítulos históricos de geometría fractal de la naturaleza es indispensable agregar un amplio volumen, compuesto en su mayoría por artículos publicados en prestigiosas revistas. En 2004 afirma sucintamente: “The most importamt results, due to the autor, consist in extensions of the Fatou-Julia theory" [14].

Mandelbrot no hizo referencia en ningún lugar de su obra expresamente a las relaciones entre fractalidad y complejidad, pero sí entre fractalidad y caos. Mi propósito aquí consiste en señalar de manera expresa, incluso contra Mandelbrot mismo, que la geometría de fractales es un modo propio de las ciencias de la complejidad y que las iteraciones fractales son operaciones mediante las cuales lo no-complejo puede ser transformado en complejidad creciente; al cabo, complejidad no-lineal, complejidad emergente, complejidad creciente, etc.

La ecuación que expresa la transformación no-lineal de un fenómeno lineal es, por tanto, la misma ecuación que produce fractalidad, válida igualmente para sistemas multifractales. Una manera en ciencia, en general, de introducir una novedad consiste en extender un modelo determinado, pre-existente.

... Every reader of mystery novels recalls many cases in which the sequence of the visits of a suspect to a house looks complex, but the sequence of the supect's displacements about town obeys simple rules. Therefore, it is not totally surprising that when one is faced with phenomena restrcited to the line, a frequently effective way to simplify cnsists in interpreting them as the trace left upon the line by the corresponding phenomena residing in the plane. Using again the peculiar vocabulary of mathematicians, many mathematical theories can be simplified by being "complexified" by which a real number $\mathrm{x}$ is changed to a complex number $\mathrm{z}$ [14]. 
Sorprendentemente, Mandelbrot [14] desarrolla la ecuación generadora de fractalidad ${ }^{8}$ pero no llega a interpretarla como transformación de la linealidad en no-linealidad, o de lo no-complejo en complejo. Huelga decir que un número complejo no tiene la misma acepción de un fenómeno de complejidad creciente en sentido estricto.

\section{Metodología}

Como se aprecia, este artículo se sitúa en el centro del trabajo con las ciencias de la complejidad, es una investigación eminentemente teórica y abarca uno de los más difíciles problemas en complejidad, sobre el cual, paradójicamente, no existe prácticamente ningún trabajo en el mundo, a saber: estudiar si, y si sí, cómo, es posible la transformación de un sistema no-complejo en uno complejo. Este artículo ha sostenido que es posible y cómo lo es. El énfasis aquí ha sido al mismo tiempo histórico y heurístico.

Luego de un trabajo sobre el estado del arte y sobre las principales fuentes de trabajo en sistemas no-lineales, el artículo ha identificado en los fractales, a partir de los trabajos de B. Mandelbrot, la condición más idónea para estudiar y resolver problema identificado. Al fin y al cabo, uno de los ejes y acaso el más importante, en el trabajo de los complejólogos consiste en complejizar los fenómenos. Eso es, de manera central, lograr que ganen grados de libertad.

La iteración fractal, una transformación habitual en topología, y acaso el fundamento mismo de las estructuras fractales, caracterizadas por autosimilitud, sostiene el artículo, es la forma más expedita de transformación de un fenómeno no-complejo a uno complejo. Por tanto, cabe decir en propiedad, a un fenómeno fractal. A fin de estudiar cómo acontece se ha tomado la ecuación generadora de fractales.

Sobre la base de haber ilustrado en qué consisten los patrones y los fenómenos de discreción, se logra demostrar la muy fuerte relación entre complejidad, no-linealidad y fractalidad. En síntesis, la metodología, para el núcleo duro del problema considerado ha sido una combinación de lógica y matemáticas, muy específicamente, de sistemas discretos.

\section{Conclusiones}

El descubrimiento de los patrones no es exclusivo de la geometría de fractales, sino, se inicia, como he querido mostrarlo, de manera puntual con Peano. Sin embargo, la geometría de fractales nos permite resolver el segundo de los dos problemas fundamentales de los sistemas complejos: la transformación de la linealidad en no-linealidad o también de la no-complejidad en complejidad.

\footnotetext{
${ }^{8}$ Una observación puntual pero importante se impone: no por ser caotólogo se es complejólogo, así como no por ser paraconsistentista se es complejólogo, por ejemplo. Pues bien, exactamente en este mismo sentido, no por trabajar fractales se trabaja necesariamente en complejidad. Pero, por el contrario, cuando se trabaja en complejidad sí se atraviesa o se puede atravesar por caos, fractales, termodinámica del no-equilibrio y demás ciencias de la complejidad. En otras palabras, sencillamente, y a título general: existen descubrimientos en la historia de la ciencia de los cuales sus propios autores no han sido conscientes. Los ejemplos abundan desde la física hasta las matemáticas, desde la biología hasta la sociología, por ejemplo.
} 
La puerta es clara y la clave, no admite dudas: se trata de la iteración fractal. Las iteraciones fractales son transformaciones mediante las cuales los sistemas/nosotros ganan/ganamos grados de libertad. La deuda inmediata es con la geometría de fractales, un fenómeno sobre el cual el propio Mandelbrot parece no haber caído en la cuenta9 ${ }^{9}$.

\section{Coda}

El descubrimiento de la transformación fractal de la linealidad en no-linealidad sufre una situación análoga a lo que, en otro plano y contexto pusiera de manifiesto A. Axelrod con respecto a la solución al Dilema del prisionero: la cooperación puede ser alcanzada gracias a juegos iterativos. Esto es, sencillamente: debido a que debemos jugar un mismo juego a largo plazo, la mejor alternativa que hay/que queda es la cooperación. Axelrod logra demostrar esta idea justamente gracias a simulaciones de juegos iterativos [16].

No en vano la teoría de juegos es uno de los elementos constitutivos de las matemáticas de sistemas discretos. Pues bien, la transformación de la linealidad en no-linealidad consiste en la transformación de un universo continuo a una comprensión discreta de la realidad. Las consecuencias de esta idea no son pocas y, sin embargo, permanecen apenas esbozadas en la historia de la ciencia en general y de las matemáticas y la geometría en particular.

\section{Agradecimientos}

Quiero agradecer, de un lado a la editora invitada de la revista por la calidad de los evaluadores que obtuvo para mi artículo y, de otra parte, al mismo tiempo quiero agradecer a los evaluadores. En particular, uno de ellos me ayudó a comprender mayor mi propio problema y mi propia tesis. Según ese concepto, una iteración lineal siempre produce un resultado lineal. Tiene absolutamente toda la razón. Pero olvida que una iteración fractal o bien admite, o bien implica no-linealidad. Es, particularmente, el caso con los fractales de Mandelbrot. Ciertamente que el caso de Peano o de Möbius no sean del caso. Pero como se desprende del marco de mi artículo, el énfasis se sitúa en la obra de Mandelbrot y sus deudas y distancias notablemente con G. Julia y P. Fatou.

\section{Referencias}

[1] S. Kauffman, Investigations. Oxford, Oxford University Press, 2000.

[2] C. E. Maldonado, Termodinámica y complejidad. Una introducción para las ciencias sociales. Bogotá, D.C. Desde Abajo [primera edición 2005], Ed. Universidad Externado de Colombia, 2011.

[3] C. E. Maldonado, "Ciencias de la complejidad: Ciencias de los cambios súbitos”, Odeón. Observatorio de Economía y Operaciones Numéricas, Universidad Externado de Colombia, 2005, pp. 85-125.

[4] D. Campos, "Caos y complejidad en el marco de cuatro revoluciones científicas", Maldonado, C. E. (Ed.), Complejidad: revolución científica y teoría, Bogotá, D.C., Ed. Universidad del Rosario, 2009, págs. 21-33.

\footnotetext{
${ }^{9}$ A título especulativo, creo que la razón por la cual Mandelbrot no se percata de la transformación fractal de la linealidad en no-linealidad tiene que ver con su asunción de que los fractales, y por derivación la complejidad, trata de sistemas continuos, cuando, en realidad, ponen de manifiesto que vivimos en un universo esencialmente probabilístico, no-ergódico y discreto. En otro trabajo aparte en curso me ocupo de este último problema, es decir: del carácter discreto del mundo, en unión con la no-ergodicidad y la contingencia.
} 
[5] A. C. Scott, The Nonlinear Universe. Chaos, Emergence, Life. Springer Verlag, 2007.

[6] E.Tiezzi, La belleza y la ciencia. Hacia una visión integradora de la naturaleza. Barcelona, Icaria, 2006.

[7] D. Zahavi, "Beyond Empathy: Phenomenological Approaches to Intersubjectivity". Journal of Consciousness Studies, 8, 2001, pp. 151-167.

[8] L. Mlodinow, Euclid's Window. The Story of Geometry from Parallel Lines to Hyperspace. New York, The Free Press, 2001.

[9] P. Bak, How Nature Works. The New Science of Self-organized Criticality. Copernicus, 1996

[10] B. Mandelbrot, La geometría fractal de la naturaleza. Barcelona, Tusquets, 1997.

[11] G. Bateson, Espíritu y naturaleza. Buenos Aires: Amorrortu, 2004.

[12] C. A. Pickover, La banda de Möbius. Todo sobre la maravillosa banda del Dr. Möbius: matemáticas, juegos, literatura, arte, tecnología y cosmología. Almuzara, 2009.

[13] F. López Aguilar y F. Brambila Paz, (Eds.). Antropología fractal. México, Sociedad Matemática Mexicana, 2007.

[14] B. Mandelbrot, Fractals and Chaos. The Mandelbrot Set and Beyond. Springer Verlag (with a Foreword by P. W. Jones and texts co-authored by C. J. G. Evertz and M. C. Gutzwiller), 2004.

[15] H. Pagels,Los sueños de la razón. El ordenador y los nuevos horizontes de las ciencias de la complejidad. Bacelona, Gedisa, 1991.

[16] R. Axelrod, The Complexity of Cooperation. Agent-Based Models of Competition and Collaboration. Princeton, NJ, Princeton University Press, 1997.

[17] T. Todorov, La conquête de l' Amérique. La question de l' autre, Paris, Seuil, 1982.

[18] B. Greene, El universo elegante: Supercuerdas, dimensiones ocultas y la búsqueda de una teoría final, Barcelona, Crítica, 2001

[19] Mandelbrot, B., (1997). La geometría fractal de la naturaleza. Barcelona: Tusquets, pág. 586.

\section{Carlos Eduardo Maldonado.}

Ph.D. en Filosofía por la KULeuven (Bélgica), post-doctorado como Visiting Scholar en la Universidad de Pittsburgh (EE.UU); postdoctorado como Visiting Research Professor en la Catholic University of America (Washing- ton, D.C.), Academic Visitor, Facultad de Filosofía, Universidad de Cambridge (Inglaterra); profesor titular, Facultad de Ciencia Política y Gobierno, Universidad del Rosario; ha sido reconocido con la "Distinción al Mérito", por la Universidad Nacional Mayor de San Marcos, Lima, Perú, por sus contribuciones a la filosofía y a la complejidad (2008); premio Portafolio, Mención de Honor Categoría Mejor Docente (2008); "Profesor Distinguido", título conferido por la Universidad del Rosario (2009); Investigador Senior (Colciencias); Senior Member-IEEE. Doctor Honoris causa, Universidad de Timisoara (Rumania), 2015. 